\title{
Switched Reluctance Machine Modeling through Multilayer Neural Networks
}

\author{
A. C. F. Mamede ${ }^{1}$, J. R. Camacho ${ }^{1}$ and R. E. Araújo ${ }^{2}$ \\ ${ }^{1}$ Department of Electrical Engineering \\ Universidade Federal de Uberlândia (UFU) \\ Campus Santa Mônica - Av. João Naves de Ávila, 2121. Postcode: 38400-902 - Uberlândia (Brazil) \\ Phone:+55 3239 4734, e-mail: anacamilamamede@gmail.com, jrcamacho@ufu.br \\ ${ }^{2}$ INESC TEC and Faculty of Engineering \\ University of Porto \\ Porto (Portugal) \\ Phone:+55 3239 4734, e-mail: raraujo@fe.up.pt
}

\begin{abstract}
The work deals with the application of artificial neural networks (ANNs) in the modeling of switched reluctance machines (SRMs). The performance of a SRM is determined by its geometry, materials used and levels of excitation. In this way, this work investigates the influence of the stator and rotor back iron thickness in the performance of SRM. A multilayer neural network is proposed to learn the nonlinear characteristics of the motor. Data of flux linkages and torque are obtained through simulations of finite elements and used for ANN training. The algorithm developed in Octave allows the user to adjust the network parameters. The results presented confirm the feasibility of using ANN to establish a predictive model of SRM performance, thus enabling further investigation in the future.
\end{abstract}

\section{Key words}

Switched reluctance machine, artificial neural networks, multilayer, modeling, predictive model, SRM performance.

\section{Introduction}

Switched Reluctance Machines (SRMs), due to its simplicity of construction and low cost has gained importance and has been considered for applications in various fields. SRM has a number of advantages: simple construction with no rotor coils, low phase tolerance, ruggedness, low production costs, high speed and variable speed applications, high temperature operation and intense temperature variations [1]. Its main disadvantages are the discontinuous torque and the ripple effect on the torque, which cause noise and vibration [2].

The traditional design model is usually composed of an iterative process in which the engineer creates a basic design using experiences and engineering skills in specific motor-specific solutions. At each stage of the project, the motor can be recalculated and the results used to modify the previous motor design. The process using Finite
Element Analysis (FEA) in 2D or 3D has many advantages over the previous process, however it takes a lot of computational time for the specific simulation [3].

As known, the inductance and torque in an SRM are nonlinear functions of phase voltage, current, rotor position and rotor speed. They are difficult to measure during operation and difficult to express with analytical functions [4].

Torque can be measured directly through a torque sensor, but requires the motor to be built and properly mounted on an axle [5]. During the design stage, the most appropriate method to obtain torque is the finite element simulation.

Some studies of estimation or analytical calculation of the torque profile have been presented to date. Chapter 2 of reference [2] is devoted to the analytical calculation of the SRM flux linkages. Artificial neural networks (ANNs) are used to model reluctance machines in [3], [4] and [6]. A nonlinear model of a synchronous reluctance motor was defined by the magnetic flux density along the air gap in [3]. In [4], the motor parameters are estimated through the application of ANN. A model for estimating concatenated flux and torque as a function of stator current and rotor position is given in [6]. Already article [5] proposes a way to calculate the torque per SRM phase using ANN to interpolate motor magnetization data, measured without blocking the rotor, thus reducing costs. The proposal of this work is to use artificial neural networks (ANNs) to estimate the flux linkages and torque produced by SRM through the variation of the two internal dimensions.

The data used for the training and verification of results are all taken from the 2D finite element simulation made using the FEMM (Finite Element Method Magnetics) software [7].

The ANN verification will be done through tests, comparing the values obtained by the finite element simulation and the values found by ANN. The times of 
each simulation are shown as well. Thus, the presented modelling is attested.

\section{Principles of SRM Operation}

The production of torque in a SRM can be explained by the elementary principle of electromechanical energy conversion. In the case of a rotating machine the increase in mechanical energy can be written in terms of the electromagnetic torque and a change in the position of the rotor, such as:

$$
\Delta W_{m}=T_{e} \Delta \theta
$$

Where $T_{e}$ is the electromagnetic torque and $\Delta \theta$ the increase in the angle of the rotor. Consequently, the electromagnetic torque is given by:

$$
T_{e}=\frac{\Delta W_{m}}{\Delta \theta}
$$

For the case of constant excitation, the increment of mechanical work done is equal to the rate of change of coenergy $W^{\prime}$, which is simply the complement of energy stored in the field. The expression for the increment of the mechanical work and calculation of the co-energy are presented in equations (3) and (4), respectively.

$$
\begin{gathered}
\Delta W_{m}=\Delta W_{t}^{\prime} \\
W_{t}^{\prime}=\int \Phi d F=\int \Phi d(N i)=\int(N \Phi) d i=\int \lambda(\theta, i) i d i=\int L(\theta, i) i d i
\end{gathered}
$$

The inductance $\mathrm{L}$ and flux linkages $\lambda$ are functions of the position of the rotor and current in equation (4). This change in the co-energy value occurs between two rotor positions, $\theta_{1}$ and $\theta_{2}$ therefore the torque in the air gap as a function of co-energy can be represented as a function of the rotor position and the excitation current.

$$
T_{e}=\frac{\Delta W_{m}}{\Delta \theta}=\frac{\Delta W_{t}^{\prime}}{\Delta \theta}=\left.\frac{\Delta W_{t}^{\prime}(i, \theta)}{\Delta \theta}\right|_{i=\text { constante }}
$$

If the inductance varies linearly with the position of the rotor for a given current, which usually does not occur in practice, then the torque can be derived as:

$$
T_{e}=\frac{d L(i, \theta)}{d \theta} \cdot \frac{i^{2}}{2}
$$

Where:

$$
\frac{d L(\theta, i)}{d \theta}=\left.\frac{L\left(\theta_{2}, i\right)-L\left(\theta_{1}, i\right)}{\theta_{2}-\theta_{1}}\right|_{i=\text { constante }}
$$

For a switched reluctance machine, the inductance is not constant and varies continuously with the position of the rotor. This has the implication that the switched reluctance motor will not have a steady-state equivalent circuit in the sense that the dc and ac motors have [2].

The equivalent circuit of the SRM must be able to explain the behavior of the SRM under all operating conditions. Such as: with and without magnetic saturation, continuous and discontinuous conduction of current, operation in the four quadrants [8]. For this, the equivalent circuit must have variable parameters, as shown in [8].

\section{ANN and Retropropagation Error}

Artificial neural networks are information-processing systems inspired by biological neural networks. These neural networks present mathematical models of an artificial neuron that aim to learn patterns based on training to take later decisions [9].

In a neural network several units called neurons are interconnected maintaining binding weights and this set forms a network. Through this network the input units are processed in the neuron and the output is determined by this processing and by an activation function. This network is able to learn patterns based on the training in which it is submitted [9]. Neural networks are used to solve problems involving pattern recognition, prediction, optimization, associative memory and control.

Multi-layer neural networks consist of a set of sensory units that constitute the input layer, one or more hidden layers of neurons and an output layer, as shown in Figure 1 [10]. The signal propagates forward through the network, layer by layer. These networks are called multilayer perceptrons, which represent a generalization of the single-layer perceptron [10].

Multiple layer networks can solve more complicated problems than single layer networks, however their training is more difficult [9]. The training is supervised through the backpropagation algorithm [10].

The backpropagation algorithm tries to find iteratively the minimum difference between the desired outputs and the outputs obtained by the neural network, with the minimum error. Thus, by adjusting the weights between the layers through the backpropagation of the error found in each iteration [10].

Basically, learning by backpropagation of error consists of two steps through the different layers of the network: a step forward, propagation, and a step backwards to retro propagation, according to Figure 2.

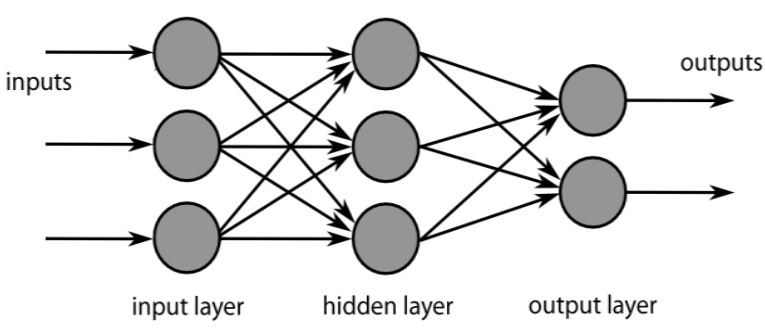

Fig. 1. Artificial neural network of multiple layers.

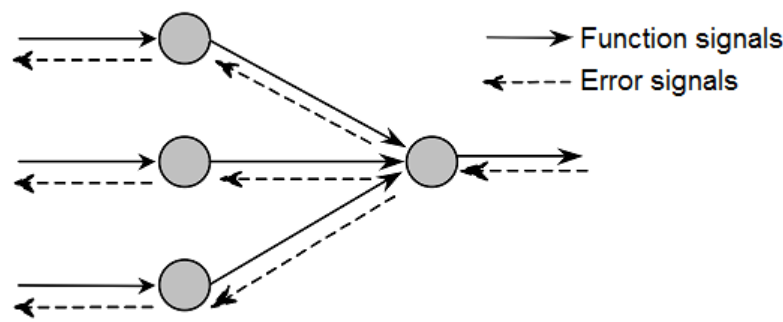

Fig. 2. Flows of the input and error signals.

In the step forward, an activity pattern (input vector) is applied to the sensory nodes of the network and its effect propagates through the network, layer by layer. Finally, an output set is produced as the actual network response. During the propagation step, the synaptic weights of the network are all fixed. During the back step, on the other hand, the synaptic weights are all adjusted according to an error correction rule. Specifically, the actual network response is subtracted from a target response to produce 
an error signal. This error signal is then propagated back through the network, against the direction of the synaptic connections - hence the name back-propagation error. The synaptic weights are adjusted to cause the actual network response to move closer to the desired response, in a statistical sense [10].

A multilayer perceptron has three distinctive features:

- The model of each network neuron normally includes a nonlinearity in its output. A sigmoidal nonlinear function is typically used.

- The network contains one or more layers of hidden neurons that are not part of the input or output of the network. These hidden neurons enable the network to learn complex tasks by progressively extracting the most significant characteristics of input patterns.

- The network exhibits a high degree of connectivity, determined by the network synapses.

\section{Model of SRM with ANN}

SRM performance, both electric and magnetic, depends on its geometric construction, materials used and level of excitation [3]. It is almost impossible to determine exact mathematical equations that take into account all these influential parameters [3]. In this way, the main idea of this work is to develop a model that incorporates the influence of some parameters and is able to give useful results to calculate the performance of the electric machine.

The dimensions of the SRM that will be varied are the stator and rotor back iron thickness, Figure 3 presents these dimensions in general SRM.

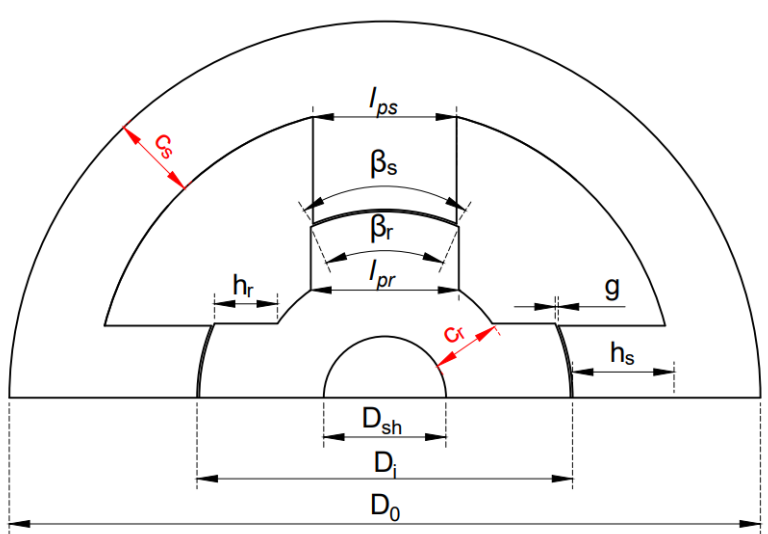

Fig. 3. Dimensions in a single-phase SRM.

In Figure 3:

$$
\begin{aligned}
& \beta_{s}=\text { stator pole arc; } \\
& \beta_{r}=\text { rotor pole arc; } \\
& l_{p s}=\text { stator pole width; } \\
& l_{p r}=\text { rotor pole width; } \\
& c_{s}=\text { stator back iron thickness; } \\
& c_{r}=\text { rotor back iron thickness; } \\
& h_{s}=\text { stator pole height; } \\
& h_{r}=\text { rotor pole height; } \\
& D_{s h}=\text { rotor shaft diameter; } \\
& D i=\text { stator inner diameter; } \\
& D_{0}=\text { stator outer diameter; } \\
& g=\text { air gap. }
\end{aligned}
$$

In the design step these values are determined from a relation with the stator pole width. The stator back iron thickness must be wide enough to support half of the flux density passing through the stator pole. Therefore, the stator back iron thickness must be at least half the width of the stator pole. However, to improve robustness and minimize vibration and noise an additional factor should be considered [2]. Thus, the value of the stator back iron thickness must be chosen in the interval presented in equation (8).

$$
\begin{gathered}
l_{p s}>c_{s} \geq 0.5 l_{p s} \\
0.5 l_{p s}<c_{r}<0.75 l_{p s}
\end{gathered}
$$

The choice of rotor back iron thickness value is based on structural integrity and operating flux density. It need not be as much as the stator back iron thickness and neither has to be equal to the minimum value equal to half the stator pole width. The range of values to be chosen from has to account for the larger interpolar air gap to provide a high ratio between the aligned and unaligned inductances, but at the same time it is desirable to have shorter rotor poles to generate minimum vibration in the rotor. Based on these considerations, the rotor back iron thickness must be determined in the interval presented in equation (9).

The motor performance parameters evaluated in this work are the flux linkage per phase, inductance and the torque per phase, these values will be obtained through a simulation of finite elements, carried out in FEMM software, for several rotor positions.

The details of the prototype studied in this work are presented in Table I. Figure 4 shows the threedimensional plot of the inductance versus position of the rotor for a variable current applied to a phase of the SRM. Figure 5 presents the torque developed by a SRM phase versus rotor position for different current values.

Table I - Motor details [2]

\begin{tabular}{|l|l|}
\hline Number of stator poles & 8 \\
\hline Number of rotor poles & 6 \\
\hline Stator pole arc & 18 degrees \\
\hline Rotor pole arc & 22 degrees \\
\hline Air gap length & $0.5 \mathrm{~mm}$ \\
\hline Outer stator diameter & $190 \mathrm{~mm}$ \\
\hline Bore diameter & $100.6 \mathrm{~mm}$ \\
\hline Stack length & $200 \mathrm{~mm}$ \\
\hline Shaft diameter & $28 \mathrm{~mm}$ \\
\hline Stator back iron thickness & $12 \mathrm{~mm}$ \\
\hline Rotor back iron thickness & $16 \mathrm{~mm}$ \\
\hline Height of stator pole & $32.7 \mathrm{~mm}$ \\
\hline Height of rotor pole & $19.8 \mathrm{~mm}$ \\
\hline Turns per phase & 154 \\
\hline Conductor area of cross section & $1,588 \mathrm{~mm}^{2}$ \\
\hline Rated current & $13 \mathrm{~A}$ \\
\hline Lamination material & M43 \\
\hline
\end{tabular}

For the rotor position equal to $30^{\circ}$, the stator and stator poles are aligned for the analyzed phase, it's when the motor experiences a higher saturation and the higher inductance values occur, see Figure 4. This value will decrease as the rotor goes to the unaligned position.

In Figure 6, the flux linkage versus rotor position and phase current is shown. The saturation of phase winding 
at high currents is clearly represented. At unaligned position, the winding presents linear behavior. At aligned position, the winding is highly saturated at rated current.

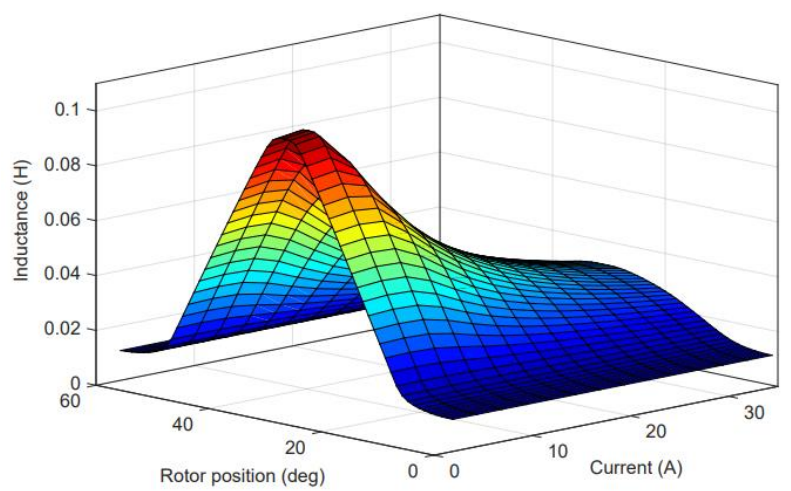

Fig. 4. Phase inductance vs. rotor position for different current values.

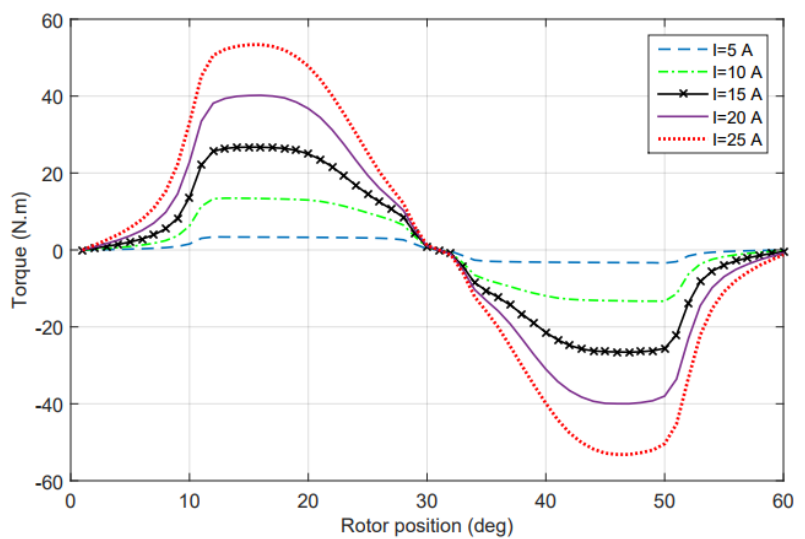

Fig. 5. Torque developed by a SRM phase vs. rotor position for different current values.

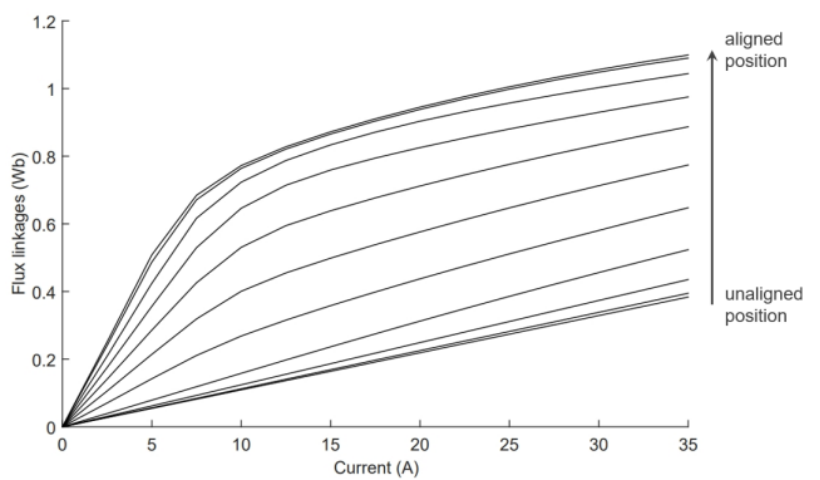

Fig. 6. Flux linkage by a SRM phase at different currents and different rotor positions.

For this motor, the back iron thickness values can be chosen in the range, as follows:

$$
\begin{gathered}
l_{p s}=D_{i n} \operatorname{sen}\left(\frac{\beta_{s}}{2}\right)=15,74 \mathrm{~mm} \\
15,74>c_{s} \geq 7.87 \mathrm{~mm} \\
7.87<c_{r}<11.8 \mathrm{~mm}
\end{gathered}
$$

The motor presented in Table I was taken from [2], and for this specific case the value of the rotor back iron thickness was $16 \mathrm{~mm}$, corresponding to $l_{p s}$, so the interval considered in this study is the same as $c_{s}$.

\section{ANN Implementation}

For this work, the rotor position, stator and rotor back iron thickness was considered as input data and the user can choose two output data to perform the training, the flux linkage and the developed torque. For each vector $\left[c_{s}, c_{r}\right]$ a finite element simulation was performed, from which the flux linkage and torque values were extracted to 60 different rotor positions. The rotor was rotated $60^{\circ}$ from 1 in 1, totaling 60 different positions. The ANN will be trained to reproduce the flux and torque curve given by FEMM.

The implemented ANN has an input layer, a hidden layer, and an output layer. The training uses the backpropagation algorithm of the error presented previously with the presence of the momentum factor. The program was implemented in Octave software, and several training tests were performed in order to adjust the network parameters appropriate to each function, flux linkage and torque.

The data acquisition for the training was done through an Octave script and FEMM simulation. The script automatically draws the SRM into FEMM, allowing you to change back iron thickness values, rotate the rotor, and save the flux and torque values for each position in a worksheet.

For the flux linkage, ANN was adjusted in 8 neurons in the hidden layer, learning rate equal to 0.2 , momentum equal to 0.5. With these parameters ANN performed the training with a quadratic error equal to 0.0397 in 21670 cycles. The trained curve and the curve obtained through FEMM are shown in Figure 7. The graph of the quadratic error by the number of cycles is shown in Figure 8 .

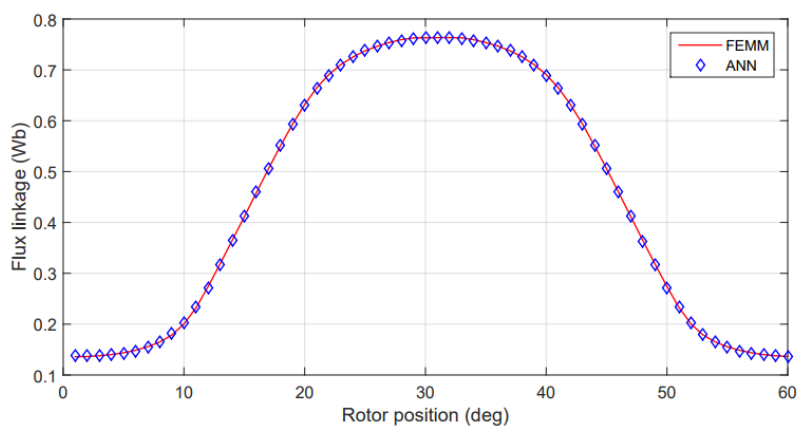

Fig. 7. Flux linkages training by the ANN.

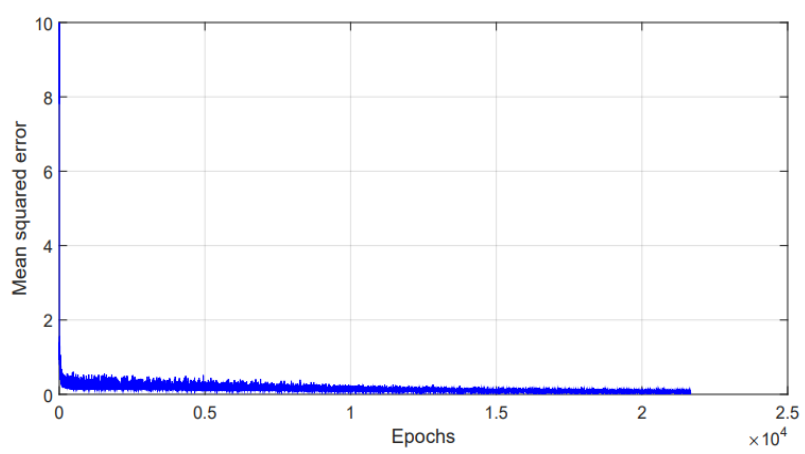

Fig. 8. Mean squared error per epochs for flux linkages training. 
As can be seen in Figure 5, approximately half the torque curve can be neglected because it corresponds to the negative part of the torque that does not exist in an actual motor prototype. For ANN training this part of the curve was neglected, because it represents a high cost of performance for the training of the network.

For the developed torque, 20 neurons in the hidden layer, learning rate equal to 0.08 and momentum equal to 0.4 resulted in a quadratic error of 0.0399 in 123645 cycles. The trained curve and the curve obtained through FEMM are shown in Figure 9. The graph of the quadratic error by the number of cycles is shown in Figure 10.

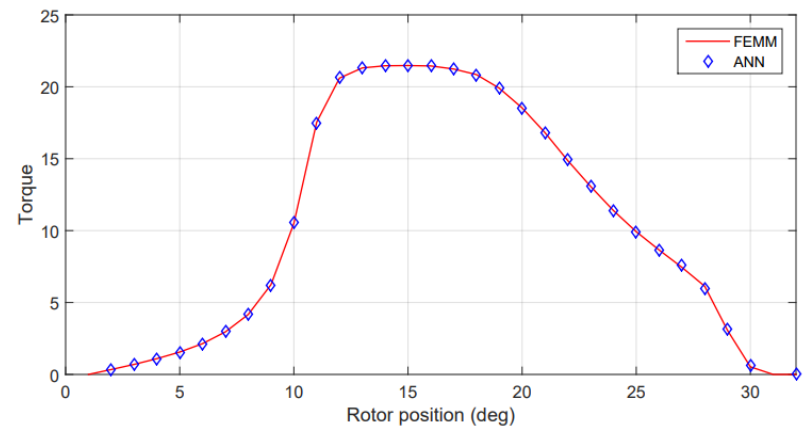

Fig. 9. Torque training by the ANN.

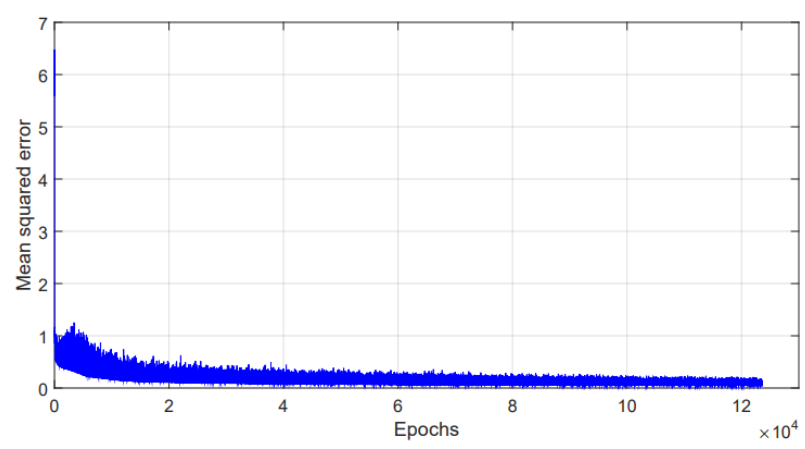

Fig. 10. Mean squared error per epochs for torque training.

\section{Modelling of SRM using ANN}

As shown in the previous section, ANN can quickly reproduce the flux and torque curves with a small error. Thus, the SRM modeling will be done through the training of several networks, so that they learn the behavior of the machine for different values of $c_{s}$ and $c_{r}$, allowing the use of these networks to estimate flux values for other back iron thickness values, avoiding the simulation of finite elements and reducing the total simulation time.

First, we performed the training of 9 vector variations $\left[c_{s}, c_{r}\right]$, comprising the upper and lower limit of equation (11) and an intermediate value. However, it was realized that only 3 variations were required for the program to satisfactorily estimate the flux value for a given pair of back iron thickness. Considering the vector $\left[c_{s}, c_{r}\right]$, the vectors $[8,8],[12,12]$ and $[16,16]$ were used for the training of 3 networks.

Figures 11 and 12 show the network-trained flux curve and the curve obtained by the FEMM for two modeling tests presented. The maximum relative error presented between the two curves was $0.4787 \%$ and $2.4587 \%$, showing that the model presented can be used to predict SRM performance in other studies.

Table II presents the comparison of the performance of the two models presented, the finite element modeling and the modeling through artificial neural networks. Computational resources: Intel Core i7 processor 6500U CPU @ 2.50GHz, 8GB of RAM.

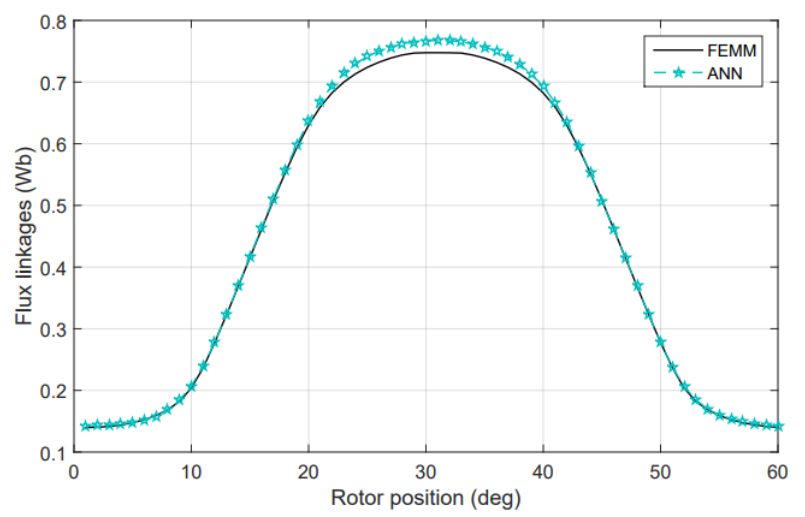

Fig. 11. Test 1: $c_{s}=11 \mathrm{~mm}$ e $c_{r}=13 \mathrm{~mm}$

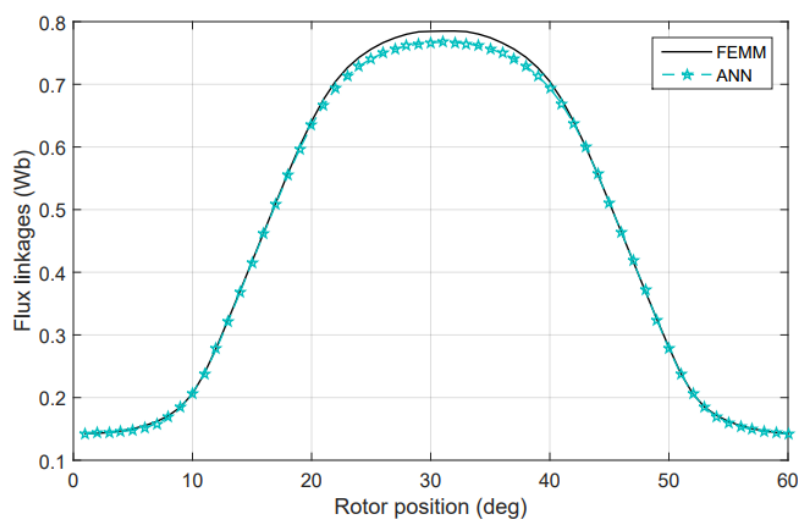

Fig. 12. Test 2: $c_{s}=13 \mathrm{~mm}$ e $c_{r}=11 \mathrm{~mm}$

Table II - Performance comparison.

\begin{tabular}{|c|c|c|c|c|}
\cline { 2 - 5 } \multicolumn{1}{c|}{} & $\begin{array}{c}\text { ANN (3 } \\
\text { networks) }\end{array}$ & FEMM & Test 1 & Test 2 \\
\hline Time (s) & 305.0286 & 850.2309 & 9.6643 & 10.0259 \\
\hline
\end{tabular}

Through Table II we can conclude that the total mean time for ANN modeling is 2855.72s, including finite element simulation and training of 3 networks. After this, the flux curve estimation time for each test was in average $10 \mathrm{~s}$, a very low value when compared to finite element simulation. Therefore, we can conclude that ANN modeling is fast and efficient, has a low error value and can be used to predict SRM performance in future studies.

\section{Conclusions}

In this work, a method of applying artificial neural networks for the modeling of the performance of switched reluctance machines was established. The ANN training data were obtained through finite element simulations using the FEMM software. The geometric construction of the SRM was made by varying the values of the rotor and stator back iron thickness, and the 
simulation output parameters were the value of the flux linkages and torque developed per phase.

The artificial neural network topology used in this work was the multilayer network, with a hidden layer and learning by backpropagation algorithm. This type of network proved adequate and the program implemented efficient to solve the proposed problem.

In comparison to finite element simulation, the ANN modeling was shown to be fast and efficient for estimating the SRM flux curve. To obtain 60 values of concatenated flow through the ANN model an average of $10 \mathrm{~s}$ is spent, by the simulation of finite elements this value was on average 85 times higher.

Thus, the model presented in this article makes it possible to use finite element simulation data in studies that require a large volume of tests. For example, SRM design optimization, study of the influence of dimensions on machine performance, among others, contributing with satisfactory results and a drastic reduction in simulation time.

\section{Acknowledgement}

The authors wish to thank the Coordination for the Improvement of Higher Education Personnel Agency (CAPES) of the Brazilian Ministry of Education for the financial resources for the development of this research work.

\section{References}

[1] J. Ahn, "Switched reluctance motor", in Torque Control, 1st ed., M. Lamchich, Ed. Intech, 2011.

[2] R. Krishnan, Switched reluctance motor drives. Boca Raton, FL: CRC Press, 2001.

[3] P. Bajec, B. Zidaric and D. Miljavec, "Artificial Neural Network Modeling of Synchronous Reluctance Motor", in Progress In Electromagnetics Research Symposium, Marrakesh, 2011, pp. 1854-1858.

[4] Lu, W., Keyhani, A. and Fardoun, A. (2003). Neural network-based modeling and parameter identification of switched reluctance motors. IEEE Transactions on Energy Conversion, 18(2), pp.284-290.

[5] F. Kucuk, H. Goto, H. Guo and O. Ichinokura, "Artificial neural network based torque calculation of switched reluctance motor without locking the rotor", Journal of Applied Physics, vol. 105, no. 7, pp. 07F103, 2009.

[6] Ustun, O. (2009). A nonlinear full model of switched reluctance motor with artificial neural network. Energy Conversion and Management, 50(9), pp.2413-2421.

[7] Meeker, D. C. (2016). Finite Element Method Magnetics. [online] Version 4.2 (12Jan2016 Build). Available at: http://www.femm.info [Accessed 14 Nov. 2017].

[8] Athani, V. and Walivadekar, V. (1994). Equivalent Circuit for Switched Reluctance Motor. Electric Machines \& Power Systems, 22(4), pp.533-543.

[9] L. Fausett, Fundamentals of neural networks, 1st ed. Upper Saddle River, N.J.: Prentice-Hall, 1994.

[10] S. Haykin and P. Engel, Redes neurais, 1st ed. Porto Alegre: Bookman, 2001. 